PHYSICAL REVIEW E 71, 031404 (2005)

\title{
Anisotropic mean-square displacements in two-dimensional colloidal crystals of tilted dipoles
}

\author{
V. A. Froltsov, C. N. Likos, and H. Löwen \\ Institut für Theoretische Physik II, Heinrich-Heine-Universität Düsseldorf, Universitätsstraße 1, D-40225 Düsseldorf, Germany \\ C. Eisenmann, U. Gasser, P. Keim, and G. Maret \\ Fachbereich Physik, Universität Konstanz, Postfach 5560, 78457 Konstanz, Germany \\ (Received 16 November 2004; revised manuscript received 7 February 2005; published 31 March 2005)
}

\begin{abstract}
Superparamagnetic colloidal particles confined to a flat horizontal air-water interface in an external magnetic field, which is tilted relative to the interface, form anisotropic two-dimensional crystals resulting from their mutual dipole-dipole interactions. Using real-space experiments and harmonic lattice theory we explore the mean-square displacements of the particles in the directions parallel and perpendicular to the in-plane component of the external magnetic field as a function of the tilt angle. We find that the anisotropy of the mean-square displacement behaves nonmonotonically as a function of the tilt angle and does not correlate with the structural anisotropy of the crystal.
\end{abstract}

DOI: 10.1103/PhysRevE.71.031404

PACS number(s): 82.70.Dd, 61.50.-f, 64.70.Dv

It is common wisdom that a one-component classical many-body system consisting of particles at constant density that interact, e.g., via a pairwise-additive repulsive inversepower potential, freezes into a periodic crystal lattice at zero temperature [1]. At finite temperatures and prior to melting, the crystal is still stable but the particles perform smallamplitude excursions from their equilibrium positions. The averaged mean-square displacement around the equilibrium lattice sites, which is a quantitative measure of these particle excursions, plays a key role in describing the bulk melting process of the crystal: the traditional Lindemann rule [2,3] states that a solid melts if the root mean-square displacement exceeds about $10 \%$ of the lattice constant. This phenomenological rule is a good estimate for melting in three spatial dimensions. In two dimensions, however, mean-square displacements are diverging [4], but fluctuations of the relative distance of nearest neighbors can be nevertheless used to establish a bulk Lindemann melting rule [5].

In this paper, we investigate the anisotropy of the meansquare displacements in two-dimensional anisotropic crystals. In high symmetry crystals, the mean-square displacement is expected to be almost isotropic. Typically, there is only a small difference in the amplitudes of the particle excursions in the direction towards their nearest neighbors and those pointing towards a void. In fact, detailed investigations for three-dimensional hard sphere face-centered-cubic crystals have revealed that the anisotropies are only significant close to melting [6]. This will change, on the contrary, for anisotropic crystals of low symmetry, where the crystal structure itself already provides different lattice constants forming the conventional unit cell. An interesting question is whether the anisotropy of the underlying crystal lattice correlates with the anisotropy of the particle mean-square displacement, i.e., whether the latter follows the crystal anisotropy or not.

Here we study an anisotropic two-dimensional colloidal crystal composed of superparamagnetic particles that are pinned by gravity to a horizontal water-air interface [5] and experience an external magnetic field $\mathbf{B}$ tilted relative to the normal of the water-air interface by an angle $\varphi$, i.e., we as- sign the value $\varphi=0^{\circ}$ to a magnetic field pointing perpendicular to the confining plane. The magnetic field induces magnetic dipoles in the colloidal particles [7] and the mutual interaction between them is a pairwise-additive dipole-dipole interaction. If the magnetic field is directed perpendicular to the interface $\left(\varphi=0^{\circ}\right)$, the in-plane interaction is isotropic and purely repulsive. Then, a two-dimensional triangular (or hexagonal) crystal is stable. By changing the tilt angle $\varphi$ of the field, the in-plane interaction becomes anisotropic and the corresponding stable crystal has the structure of a twodimensional oblique lattice $[8,15]$. We explore the anisotropy of the mean-square displacements in both directions, perpendicular and parallel to the in-plane projection $\mathbf{B}_{\|}$of the applied external field $\mathbf{B}$, as a function of the tilt angle $\varphi$ in the regime where the interactions are anisotropic but still repulsive. Our main finding is that the anisotropy of the meansquare displacement does not correlate with the lattice anisotropy. In particular, as the tilt angle $\varphi$ is increased, the displacements towards the nearest neighbors are first smaller than those perpendicular to the field but then they exceed the latter at a tilt angle $\varphi \simeq 22^{\circ}$. This is a hint to different pathways of defect formation at higher temperatures and therefore could imply different scenarios of two-dimensional melting of anisotropic crystals [9-11]. Furthermore, both displacements behave nonmonotonically as functions of $\varphi$ at fixed temperature. We obtain our results by using both realspace experiments of magnetic colloids and a harmonic lattice theory for a dipole-dipole interaction and find good agreement between the two.

In our experiments, monodisperse polystyrene particles [12] with a diameter of $4.5 \mu \mathrm{m}$ and a mass density of $1.5 \mathrm{~g} / \mathrm{cm}^{3}$ are confined to the water/air interface of a hanging water droplet by gravity. The particles are superparamagnetic due to doping with $\mathrm{Fe}_{2} \mathrm{O}_{3}$ nanoparticles, and, therefore, magnetic dipole moments can be induced by applying an external magnetic field B. As shown in Ref. [13], the interaction between the particles is precisely described by the dipole-dipole interaction, which dominates all other interactions. The cylindrical sample cell (diameter of $8 \mathrm{~mm}$ ) is horizontally aligned, and the flatness of the interface is con- 


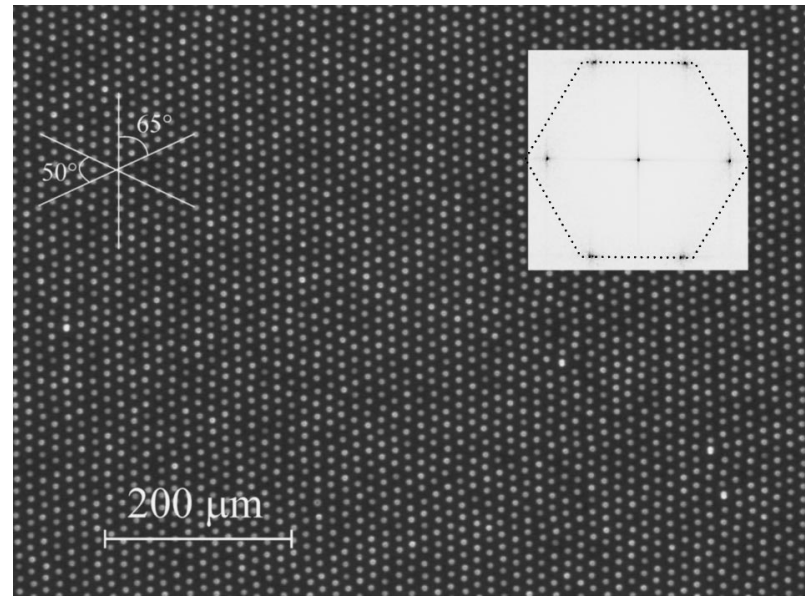

FIG. 1. Camera image of an anisotropic colloidal crystal at $\varphi$ $=21.8^{\circ}, B=0.8 \mathrm{mT}$, and $\rho=6.8 \times 10^{9} \mathrm{~m}^{-2}$. In the upper left corner nearest neighbor bond orientations are highlighted by lines. The angles of $50^{\circ}$ and $65^{\circ}$ between these directions clearly show the anisotropy of the lattice. The anisotropy is also obvious from the Fourier transformation of the particle positions (inset): There is a clear deviation from the symmetric hexagon corresponding to the triangular lattice.

trolled with an accuracy of $\pm 1 \mu \mathrm{m}$ in order to achieve a uniform particle density throughout the sample cell. The colloids were observed through a $5 \times$ microscope objective by digital video microscopy. The size of the field of view was $840 \times 620 \mu \mathrm{m}^{2}$; usually 2000-3000 particles were observed, while the whole cell contained roughly 200000 particles. The diameter of the particles is typically around 9 pixels with one pixel covering the area of roughly $1 \mu \mathrm{m}^{2}$ leading to an expected accuracy in the particle coordinates of about $\pm 0.1 \mu \mathrm{m}$. All measurements were carried out at room temperature. The magnitude of the external magnetic field $B$ was used to control the interaction strength $\Gamma$ to be defined shortly. The anisotropy of the interaction between the particles was controlled by tilting the magnetic field $\mathbf{B}$ away from the direction vertical to the sample plane.

In a system interacting by means of a power-law potential, all structural and thermodynamic properties depend on a single dimensionless combination of temperature and density, the so-called coupling constant $\Gamma$. For the case at hand, in which the magnetic moments of the involved particles are proportional to the product of the magnetic susceptibility $\chi$ and the external field $\mathbf{B}$, this coupling constant is given by

$$
\Gamma=\frac{\mu_{0}(\chi B)^{2}}{8 \pi k_{B} T} \rho^{3 / 2},
$$

where $k_{B}$ is Boltzmann's constant, $\mu_{0}$ is the magnetic perme- ability of vacuum, and $\rho$ is the two-dimensional area density of the system [14].

After equilibration of the system for several days in the crystalline state, the entire sample consisted of one monocrystalline domain. Figure 1 shows a typical example of the centered rectangular crystal lattice observed. The underlying lattice was determined by extracting the lattice constants from the Fourier transform of the particle positions and has been shown to agree perfectly with theoretical calculations [15]. Only in the case of tilting angles between $0^{\circ}$ and $10^{\circ}$ it is difficult to compare theory and experiments since within this region anisotropy is too small to align the crystal lattice with respect to the external magnetic field.

From the experimentally determined particle positions, we have extracted the projections of the time-dependent Lindemann parameter, defined as $[15,16]$ :

$$
\gamma_{x}(t)=\frac{1}{a^{2} M} \sum_{m=1}^{M}\left\langle\left[\Delta x_{j}(t)-\Delta x_{j+m}(t)\right]^{2}\right\rangle
$$

and

$$
\gamma_{y}(t)=\frac{1}{a^{2} M} \sum_{m=1}^{M}\left\langle\left[\Delta y_{j}(t)-\Delta y_{j+m}(t)\right]^{2}\right\rangle
$$

perpendicular and parallel to the in-plane component of the magnetic field, which is pointing along the $y$-axis. In Eqs. (2) and (3) above, $\Delta x_{k}(t)=x_{k}(t)-x_{k}(0)$ and $\Delta y_{k}(t)=y_{k}(t)-y_{k}(0)$ denote, respectively, the $x$ - and $y$-components of the displacement of the particle centered at the $k$ th lattice site. Moreover, the summation contains the relative displacements of $M$ neighbors of any given site and the average over those is taken via a division through $M$. Finally, the Lindemann parameter is rendered dimensionless by dividing through $a^{2}$, where $a$ is the lattice constant along the $y$-direction. In the case of an isotropic interaction potential $\left(\varphi=0^{\circ}\right)$, the particle coordinates are rotated prior to the calculation of the Lindemann parameter in order to align one of the lattice vectors with the $y$-axis, which is determined by the geometry of the magnetic field apparatus. Such a rotation is not necessary for the centered rectangular crystals $\left(\varphi>0^{\circ}\right)$, since the shorter of the two lattice vectors is then pointing along the in-plane component of the magnetic field.

In Fig. 2 we show three examples of the two projections of the Lindemann parameter for different $\Gamma$ and tilting angles. As expected for a crystalline state, a constant value is approached in the long-time limit. Note that in the case of $\varphi=21.8^{\circ}$ (Fig. 2, right) the projection parallel to the in-plane component of the magnetic field is larger than the one perpendicular to the field, whereas in case of $\varphi=19.3^{\circ}$ (Fig. 2, left) it is the other way around.
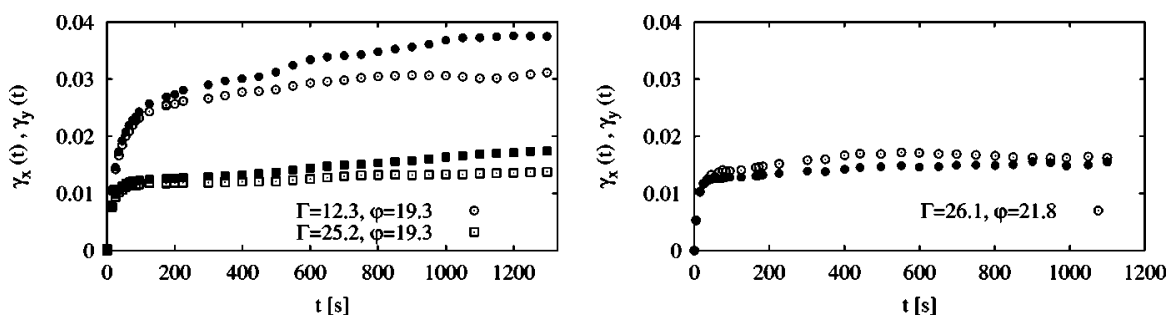

FIG. 2. The Lindemann parameter parallel (open symbols) and perpendicular (full symbols) to the in-plane component of the magnetic field for $\varphi=19.3^{\circ}$ (left) and $\varphi=21.8^{\circ}$ (right). 
In order to analyze the particle displacements theoretically, we employ harmonic lattice theory [17]. The latter provides an explicit expression for the equal-time correlators between the particle displacements, which can then be compared with the corresponding experimental long-time limit of the Lindemann parameters.

We aim to provide a theoretical explanation for the anisotropy of the long-time Lindemann parameters observed in the experiments. The Lindemann parameters along and perpendicular to the magnetic field are defined by Eqs. (2) and (3). The underlying static crystal is spanned by two lattice vectors $\mathbf{a}$ and $\mathbf{b}$ and can be equivalently described by the two lattice constants $a$ and $b$ and the angle $\psi$ between the two lattice vectors. The parameters $a, b$, and $\psi$ are uniquely determined for any given $\varphi$ : the optimal periodic structure for a given tilt angle is obtained by minimization of the groundstate energy (lattice sums) using the dipole-dipole pair interaction potential [8]:

$$
v(\mathbf{r})=\frac{\mu_{0}(\chi B)^{2}}{8 \pi} \frac{1}{r^{3}}\left(1-3 \sin ^{2} \varphi \cos ^{2} \theta\right),
$$

where $\mathbf{r}$ is the interparticle separation vector, $r$ is its magnitude, and $\cos \theta=\mathbf{r} \cdot \mathbf{B}_{\|} /\left(r B_{\|}\right)$. As it has been found in Ref. [8], the shortest of the two lattice vectors is always pointing along $\mathbf{B}_{\|}$. We adopt the convention that this vector is $\mathbf{a}$, hence $a \leqslant b$.

The harmonic theory used to calculate the equal-time correlation functions of Eqs. (2) and (3), $\left\langle\left|\Delta x_{j}-\Delta x_{j+m}\right|^{2}\right\rangle$ and $\left\langle\left|\Delta y_{j}-\Delta y_{j+m}\right|^{2}\right\rangle$ [17] is based on the diagonalization of the dynamical matrix $\mathbf{D}(\mathbf{q})$ (a two-by-two matrix in our case), obtained as the discrete Fourier transform of the real space dynamical matrix $\mathbf{D}(\mathbf{R})$ with elements [1]:

$$
D_{\mu \nu}(\mathbf{R})=\delta_{\mathbf{R}, 0} \sum_{\mathbf{R}^{\prime}} v_{\mu \nu}\left(\mathbf{R}^{\prime}\right)-v_{\mu \nu}(\mathbf{R}),
$$

where

$$
v_{\mu \nu}(\mathbf{r})=\frac{\partial^{2} v(\mathbf{r})}{\partial r_{\mu} \partial r_{\nu}}
$$

$r_{\mu, \nu}$ is the $(\mu, \nu)$-component of the vector $\mathbf{r}, \mu, \nu=x, y$, and the potential $v(\mathbf{r})$ is specified in Eq. (4). The quantities $\mathbf{R}$ and $\mathbf{R}^{\prime}$ are lattice vectors.

The diagonalization yields for every $\mathbf{q}$-value two eigenvalues $\lambda_{i}$ and the corresponding eigenvectors $\mathbf{e}_{i}, i=1,2$ with Cartesian components $e_{i, x}$ and $e_{i, y}$ on the $x$ - and $y$-axes, respectively. The Lindemann parameters in the directions perpendicular and parallel to the in-plane projection of the external magnetic field are given within this approximation through the expression [18]:

$$
\begin{gathered}
\gamma_{\mu}=\frac{k_{B} T}{K} \int_{\mathcal{C}_{r}} \frac{1}{M} \sum_{m=1}^{M} \sin ^{2} \frac{\mathbf{q} \cdot \mathbf{R}_{m}}{2}\left(\frac{e_{1, \mu}^{2}}{\lambda_{1}(\mathbf{q})}+\frac{e_{2, \mu}^{2}}{\lambda_{2}(\mathbf{q})}\right) d^{2} q \\
(\mu=x, y) .
\end{gathered}
$$

In Eq. (7) above, the integration is carried out over the first Brillouin zone $\mathcal{C}_{r}$ of the reciprocal lattice. The quantity at the denominator of the prefactor is given by $K=\pi^{2} a^{2} / A$

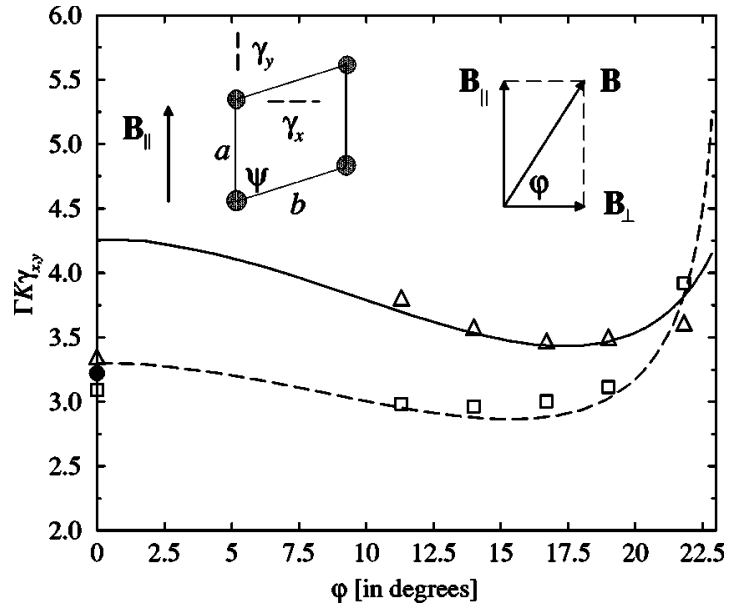

FIG. 3. The Lindemann parameters $\gamma_{x}$ and $\gamma_{y}$ plotted against the tilt angle $\varphi$ of the magnetic field. The solid (dashed) lines correspond to theoretical $\gamma_{x}\left(\gamma_{y}\right)$ while the triangles (squares) correspond to experimentally measured values, respectively. The continuous lines pertain to results obtained by keeping the two nearest neighbors in the $y$-direction in the summation of Eq. (8). The filled circle at $\varphi=0^{\circ}$ denotes the theoretical result obtained when the sum of Eq. (8) extends over all $M=6$ neighbors in the triangular lattice, which is the stable crystal structure there. Here, theory yields results for $\gamma_{x}$ and $\gamma_{y}$, which are so close to each other that cannot be resolved within symbol sizes used in the figure. We plot the values $\Gamma K \gamma_{x, y}$ which are universal within harmonic theory, whereas the experimental results were obtained for different values of $\Gamma$, varying between 10 and 27. The inset shows the geometry of the lattice and directions of the external magnetic field as well as the orientation of Lindemann parameters with respect to the lattice and field.

$\cong 10$, where $A$ is the area of the direct lattice cell, hence this ratio depends only on the tilt angle of the external field. The summation is carried out over $M$ nearest neighbors that are connected to a given lattice point through the vector $\mathbf{R}_{m}$. For the cases of tilted external fields $\left(\varphi \neq 0^{\circ}\right)$, in which the resulting crystal lattice is strongly anisotropic, the summation extended to the $M=2$ nearest neighbors. For perpendicular external field $\mathbf{B}\left(\varphi=0^{\circ}\right)$, the equilibrium lattice is triangular and hence the sum covered the $M=6$ nearest neighbors of that structure. It is useful, at this point, to introduce the rescaled, dimensionless variables $\mathbf{k}=\mathbf{q} a, \mathbf{S}_{m}=\mathbf{R}_{m} / a$ and $\xi_{i}$ $=a^{2} \lambda_{i} /\left(\Gamma k_{B} T\right)$. Then, Eq. (7) takes the form:

$$
\begin{aligned}
\gamma_{\mu}= & \frac{1}{\Gamma K} \int_{\mathcal{D}_{r}} \frac{1}{M} \sum_{m=1}^{M} \sin ^{2} \frac{\mathbf{k} \cdot \mathbf{S}_{m}}{2}\left(\frac{e_{1, \mu}^{2}}{\xi_{1}(\mathbf{k})}+\frac{e_{2, \mu}^{2}}{\xi_{2}(\mathbf{k})}\right) d^{2} k \\
& (\mu=x, y),
\end{aligned}
$$

with the integration carried over the rescaled Brillouin zone $\mathcal{D}_{r}$. The integral on the right-hand side of Eq. (8) above depends only on the tilt angle $\varphi$.

The theoretical prediction states that the Lindemann parameters scale as $\gamma \propto 1 / \Gamma$, which is the asymptotically exact limit for small temperature or high number density. The scaled anisotropic mean-square displacements as obtained from harmonic lattice theory are plotted in Fig. 3, where they are also compared to the experimental data. On the basis of 
these results, we can draw the following conclusions: First, there is very good quantitative agreement between theory and experiment at all tilt angles. Since the experimental data were taken at different values for $\Gamma$, thereby the $1 / \Gamma$-scaling as well as the dependence on the tilting angle is proven to agree with the theory. Secondly, the behavior of the meansquare displacements as a function of $\varphi$ is nonmonotonic. For increasing $\varphi$, they first decrease and then grow larger again. Third, for $\varphi<22^{\circ}$, the displacements parallel to the in-plane component of the external magnetic field are smaller than those perpendicular to this direction but the trend is reversed as the tilt angle grows to higher values. This behavior is unexpected at a first glance but can be understood by an intuitive argument; for $\varphi \approx 0^{\circ}$, there is less repulsion of the particles in the direction parallel to the field, resulting thereby in a smaller lattice constant in this direction. This, in turn, leaves less freedom for a particle to perform displacements than in the other, perpendicular direction. This finding implies that in case of the anisotropic crystals the anisotropy of the Lindemann parameter does not scale with the anisotropy of the underlying crystal lattice; the reduction of the repulsions in the $\mathbf{B}_{\|}$-direction does not automatically imply that the oscillations in this direction are broader in amplitude than in the perpendicular one. In addition, the mean-square displacements cannot simply be scaled away by relating them to the nearest-neighbor distances, as has been shown for the liquid and hexatic phase (cf. Ref. [15]). Finally, for $\varphi \approx 22^{\circ}$, the interaction is very weakly repulsive in the $\mathbf{B}_{\|}$-direction, hence a soft mode starts approaching in this direction, corresponding to stronger delocalization of particles. At $\varphi \approx 22^{\circ}$ the softness is sufficient in order to bring about a crossover between the relative magnitudes of $\gamma_{x}$ and $\gamma_{y}$ : whereas for $\varphi<22^{\circ}$ we have $\gamma_{x}>\gamma_{y}$, for $\varphi>22^{\circ}$ the reverse is true. Theory and experiment are in agreement regarding the location of this crossover behavior, as can be seen from Fig. 3. In fact, theory predicts a rapid growth of the parameter $\gamma_{y}$, which indicates that the crystal structure itself is getting mechanically unstable, as has been shown in Ref. [9].

Let us now turn our attention to the Lindemann parameters for the case of perpendicular fields, $\varphi=0^{\circ}$. As can be seen in Fig. 3, in this case the anisotropy between the $x$ - and $y$-directions almost disappears. The experimental data points for the two Lindemann parameters lie within $5 \%$ of each other, whereas the theoretical results are much closer to each other, so that only one point (the full circle) can be shown there; the difference between $\gamma_{x}$ and $\gamma_{y}$ is smaller than the symbol size. The strong reduction of the anisotropy in the Lindemann parameters is a direct consequence of the much higher isotropy of the triangular crystal, as opposed to the oblique ones, stable for $\varphi \neq 0^{\circ}$. Yet, there are two points that must be emphasized here: first, even in the triangular crystal there is a structural anisotropy between the $y$-direction (which points towards the nearest neighbors of a given particle) and the $x$-one, which points towards the voids, hence the resulting isotropy in the Lindemann parameters is not a trivial result. And secondly, in order to calculate (in theory) or measure (in experiment) Lindemann ratios that are almost equal in the $y$ - and $x$-directions, a summation over all six neighbors must be carried out. Indeed, as can be seen in Fig. 3 , if a sum over only the two neighbors in the $y$-direction (which are the nearest ones for the case $\varphi \neq 0^{\circ}$ ) is carried out, then the Lindemann parameters remain highly anisotropic all the way down to $\varphi=0^{\circ}$. This explains both the partial restoring of isotropy at $\varphi=0^{\circ}$ and the "jump" of the Lindemann values there.

In conclusion, we have demonstrated, by theory and experiment, that particle displacements in a two-dimensional anisotropic crystal are strongly anisotropic. Depending on the pair interaction between the particles, the anisotropy in the displacements does not necessarily follow the anisotropy of the lattice constants. This result was obtained for colloids but is directly relevant for any other strongly confined dipolar sheets, e.g., monolayer ice in confinement in the absence [19] or in the presence [20] of an aligning electric field. It would be interesting to measure the full phonon spectra, in analogy to what has already been done for the isotropic case [21]. Furthermore, defect formation should be included in the theory, to incorporate effects close to crystal melting. Finally it would be interesting to study inhomogeneous field effects which may give rise to crystalline clusters [22] and study the particle displacements in a finite cluster.

This work has been supported by the Deutsche Forschungsgemeinschaft (DFG), within subprojects $\mathrm{C} 2$ and C3 of the SFB-TR6 Collaborative Research Center "Physics of colloidal dispersions in external fields." We thank R. Blaak for helpful discussions.
[1] N. W. Ashcroft and N. D. Mermin, Solid State Physics (Holt Saunders, Philadelphia, 1976).

[2] F. A. Lindemann, Phys. Z. 11, 609 (1910); see also, A. R. Ubbelohde, The Molten State of Matter (Wiley, Chichester, 1978).

[3] H. Löwen, Phys. Rep. 237, 249 (1994).

[4] J. Fröhlich and C. Pfister, Commun. Math. Phys. 81, 277 (1981)

[5] K. Zahn and G. Maret, Phys. Rev. Lett. 85, 3656 (2000).

[6] R. Ohnesorge, H. Löwen, and H. Wagner, Europhys. Lett. 22, 245 (1993).
[7] J. D. Jackson, Classical Electrodynamics (Wiley, New York, 1975).

[8] V. A. Froltsov, R. Blaak, C. N. Likos, and H. Löwen, Phys. Rev. E 68, 061406 (2003).

[9] C. Eisenmann, U. Gasser, P. Keim, and G. Maret, Phys. Rev. Lett. 93, 105702 (2004).

[10] J. Toner and D. R. Nelson, Phys. Rev. B 23, 316 (1981).

[11] S. Ostlund and B. I. Halperin, Phys. Rev. B 23, 335 (1981).

[12] Dynabeads M-450 Epoxy from www.dynalbiotech.com.

[13] K. Zahn, J. M. Mendez-Alcaraz, and G. Maret, Phys. Rev. Lett. 79, 175 (1997). 
[14] Note that this definition differs from the one given in Ref. [5] by a factor of $2 \pi^{1.5} \approx 11.1$. Therefore, using this definition the phase transition from solid to hexatic occurs at $\Gamma \approx 5$.4. Moreover, for simplicity the effects of the anisotropy onto the inverse system temperature as elaborated in Ref. [15] were not taken into account.

[15] C. Eisenmann, P. Keim, U. Gasser, and G. Maret, J. Phys.: Condens. Matter 16, S4095 (2004).

[16] K. Zahn, R. Lenke, and G. Maret, Phys. Rev. Lett. 82, 2721 (1999).

[17] M. Born and K. Huang, Dynamical Theory of Crystal Lattices
(Oxford University Press, London, 1966); A. A. Maradudin, E. W. Montroll, G. H. Weiss, and I. P. Ipatova, Theory of Lattice Dynamics in the Harmonic Approximation (Academic, New York, 1971).

[18] G. Goldoni and F. M. Peeters, Phys. Rev. B 53, 4591 (1996). [19] R. Zangi and A. E. Mark, Phys. Rev. Lett. 91, 025502 (2003). [20] R. Zangi and A. E. Mark, J. Chem. Phys. 120, 7123 (2004).

[21] P. Keim, G. Maret, U. Herz, and H. H. von Grünberg, Phys. Rev. Lett. 92, 215504 (2004).

[22] V. A. Froltsov, C. N. Likos, and H. Löwen, J. Phys.: Condens. Matter 16, S4103 (2004). 